\title{
Geometric optimization of shells
}

\author{
P. Brož \\ Czech Institution of Structural and Civil Engineers, Czech Republic
}

\begin{abstract}
The selection of forward-looking evolution philosophies for the dynamic optimization of slender shell structures subject to load impact is handled. The wave mechanics in identification outlook is applied to numerical analysis of the problem.

Employing the force method of analysis and provisionally neglecting the implicit compatibility conditions, an approximate explicit problem is presented. After solving this problem a lower limit of the optimum is effectively obtained. To assess the real optimum of the implicit problem, the compatibility conditions are taken into account for the final geometry. Several approximation concepts are proposed for the effective solution of the explicit fixed geometry problem. Linear programming models and approximate treatment of the displacement constraints are presented. The proposed algorithms do not involve multiple implicit analyses of the construction.

Keywords: constraint, dynamic optimization, large span construction, structural parameters, variable linking.
\end{abstract}

\section{Introduction}

In modern structural engineering, it is important to deal with some dynamic stresses of large span slender shells. Concurrently, the methods of identification and optimization are used, e.g. in compliance with Tesár [1] and Rechenberg [2]. By means of mathematical simulations worked out we can get well operating observes appraising the physical state inclusive of optimization of the shell structure by employing measurements at few points only. As a rule, several lower natural frequencies are studied. Along these lines, a smart real time monitoring can serves for the assessment of the maximum response of such shell systems subject to severe dynamic shocks. The response cannot be measured at every critical point for some parts of the construction may be not available or a 
comprehensive instrumentation would be too costly. The control theory presents so called state observes that estimate the condition of a system by applying the measurements in combination with mathematical modelling of the roofs. Experimental, theoretical and numerical evaluations for identification and optimization of slender shell construction investigated have been lately made the focal point of intensive endeavours owing to insistent problems of disaster prevention of such structures. A sophisticated analysis of the shell systems is required so as to solve the problems being connected with the identification and optimization when subjected to the impacts.

The measured parameters are employed to better the simulation by modification of some structural parameters. The object of this paper is to identify, tune and optimize the stated models. A way to be in charge of the mentioned problems effectively is the adjustment of the wave techniques using modified mesh refinements and substructuring simulation procedures. In so doing, numerical techniques embrace the mesh modelled by moving elements.

\section{Structural optimization}

The demands for the application of the conceptions of the synthesis of structural geometry have become evident. For such structures the weight minimization or design of highly loaded construction are of extra significance. The synthesis of discrete approaches developed until now in structural mechanics has provided all necessary tools for efficient adoption of optimization procedures in the design and assessment of such structures.

Structural optimization is accepted as the selection of design parameters allowing the minimum weight or fully stressed design of structures. The selection of design parameters is subjected to the types of constraints, as follows:

- geometric constraints - minimum and maximum areas, dimensions and stiffnesses of the shell structural elements selected,

- $\quad$ stress c. - maximum allowable stress,

- displacement c. - minimum and maximum deformations, rotations and displacements,

- $\quad$ stability, fatigue and resonance constraints.

Such entries characterize the regional constraints and are applied for all load conditions possibly appearing. They are represented by constraint hypersurfaces. Stress and deformations as well as the resonance, stability and fatigue limits are, in general, the nonlinear functions of the design variables adopted. Likewise, the constraint hypersurfaces are the nonlinear functions of such variables. When the design point is located in the area above the constraint surfaces, the characteristic stress or displacements in the regional constraints will lie within the specified limits. The point where the constant-weight hypersurface touches the constraint hypersurfaces adopted is the point for the minimum weight design e.g. of bionics shell configurations investigated.

When constraints are imposed only on the stress, an iterative procedure is used to redesign the shell structure so that each element reaches limiting stress under at least one of the load conditions assumed during aimed snow slip 
loading. Such design is described as a fully stressed design. The design variables for fully stressed design converge to a vertex of $n$ hypersurfaces representing the $n$ constraints on the stresses.

The total amount of design variables, particularly in large shell roof structures, is often numerous. In many synthesis methods the solution efficiency is highly dependent on the number of variables optimized simultaneously. It is desired to reduce this number. While the optimal design is usually improved by considering geometric variables, the objective functions of minimum-weight or fully stressed designs are frequently not responsive to variations in these variables in the proximity of the optimum.

Generally, it is possible to express the optimization problem in the following way: Determine the geometric design variables $Y$ and the cross-sectional design variables $X$ or $Z$ (as other types of variables) such that during the snow slip their holds

$\begin{array}{ll}\text { objective function } & W=f(X, Y, Z) \rightarrow \min , \\ \text { geometric constraints } & X^{\mathrm{L}} \leq X \leq X^{\mathrm{U}}, \\ & Y^{\mathrm{L}} \leq Y \leq Y^{\mathrm{U}}, \\ & Z^{\mathrm{L}} \leq Z \leq Z^{\mathrm{U}}, \\ \text { stress constraints } & \sigma^{\mathrm{L}} \leq \sigma \leq \sigma^{\mathrm{U}}, \\ \text { displacement constraints } & r^{\mathrm{L}} \leq r \leq r^{\mathrm{U}}, \\ \text { resonance, stability and fatigue constraints } & \gamma^{\mathrm{L}} \leq \gamma \leq \gamma^{\mathrm{U}},\end{array}$

where $\mathrm{L}$ und $\mathrm{U}$ denote superscripts indicating lower and upper limits, respectively.

The symbols $\sigma, r$ and $\gamma$ are the selected vectors of stress displacements and resonance, stability or fatigue limits. Such bounds represent implicit functions of the design variables.

The intent of the straightforward optimization method is to solve concurrently all the above variables by one of available nonlinear programming techniques. A possible two-level solution procedure for such optimization is given by the following steps:

1. Suppose an initial structural geometry.

2. Optimize cross-sectional variables and forces for given geometry by satisfying eqns. 1-7.

3. Alter the geometric variables.

4. Repeat the operations 2 and 3 until optimal structural geometry for dangerous snow slip loading is obtained.

The quantity of design variables can be reduced by rendering all geometric dimensions via a small number of independent variables. The design variable link is often indispensable in view of such entries as functional requirements, production restrictions, boundary conditions, load parameters in the course of snow slip and so forth. Another possibility to reduce the number of candidate geometries is to use a coarse grid in the space of geometric variables, so that only a small number of $X, Y$ or $Z$ values is to be considered. This is justified in many cases, where the objective function (minimum weight design, fully 
stressed design, etc.) is not sensitive to the changes in geometric variables nearby the optimum. For the purpose of optimizing the $X, Y$ and $Z$ variables at this step, the unconstrained minimization procedure is to be applied, e.g. in compliance with Kirsch [3].

\section{Set geometrical problem}

When analyzing the approximate explicit problem by dint of the two-level concept, both the cross-sectional variables and the force distribution in the construction are optimized for any geometry. This pass that has to be repeated many times, for the most part embraces much computational endeavour and it is fundamental to utilize effective solution techniques.

One can assume that both the objective function $\mathrm{Z}$ and the matrix $[\mathrm{C}]$ are linear functions of $\{X\}$ and taking into account only the stress and side constraints, we obtain the following linear programming problem (LPP): find $\{X\}$ and $\{N\}$ such that

$$
\begin{gathered}
Z=\{l\}^{\mathrm{T}}\{X\} \rightarrow \min \\
{[X]\left\{\sigma^{\mathrm{L}}\right\} \leq\left\{A_{L}\right\}+\left[A_{N}\right]\{N\} \leq[X]\left\{\sigma^{\mathrm{U}}\right\}} \\
\left\{X^{\mathrm{L}}\right\} \leq\{X\} \leq\left\{X^{\mathrm{U}}\right\}
\end{gathered}
$$

where $\{l\}$ is a vector of constant coefficients (members length, e.g.), and $[X]$ is a diagonal matrix containing linear functions of the design variables on its principal diagonal. This statement is frequently employed in optimal plastic design of framed construction. The principal asset is that the LPP can be efficiently solved using standard computer programs. The assumption of linear dependence between $Z,[C]$ and the cross-sectional design variables $\{X\}$ is usually valid for truss structures. It is approximate for other types of framed structures such as beams and frames, however, it has been noted that the inaccuracy involved in this assumption is minor. A more serious drawback is that the displacement constraints are not considered in the linear programming solution. Some simplifications serve to include the latter constraints in the LPP formulation.

\subsection{Lagrange multipliers}

One method is to analyze first the LPP and later to control if the displacement constraints are satisfied. If a certain constraint $D_{j} \leq D_{j}^{\mathrm{U}}$ is violated, we may calculate optimal values of $X_{i}(i=1, \ldots, n)$ for which

$$
D_{j} \leq D_{j}^{\mathrm{U}} \text {. }
$$

Inserting the expression for the displacements from the general formulation into eqn (11), the problem to be solved is: find $\{X\}$ such that

$$
Z=\sum_{i=1}^{n} l_{i} X_{i} \rightarrow \min
$$




$$
\sum_{i=1}^{n} \frac{B_{j i}}{X_{i}}=D_{j}^{U}
$$

Defining the Lagrangian function

$$
\varphi=\sum_{i=1}^{n} l_{i} X_{i}+\lambda\left(\sum_{i=1}^{n} \frac{B_{j i}}{X_{i}}-D_{j}^{U}\right)
$$

the conditions that must be satisfied at the optimum are

$$
\begin{gathered}
\frac{\partial \varphi}{\partial \lambda}=\sum_{i=1}^{n} \frac{B_{j i}}{X_{i}}-D_{j}^{U}=0 \\
\frac{\partial \varphi}{\partial X_{h}}=l_{h}-\lambda \frac{B_{j h}}{X_{h}^{2}}=0 \quad h=1, \ldots, n .
\end{gathered}
$$

Solving for $\lambda$ and $X_{h}$ yields

$$
\begin{gathered}
\sqrt{\lambda}=\frac{1}{D_{j}^{U}} \sum_{i=1}^{n} \sqrt{\left(B_{j i} l_{i}\right)} \\
X_{h}=\sqrt{\lambda} \sqrt{\left(B_{j h} / l_{h}\right)} \quad h=1, \ldots, n .
\end{gathered}
$$

The demanded values of $X_{h}$ may be computed for all displacement constraints and the largest value of each design variable (considering both the displacement constraints and the values obtained by the LLP solution) is selected for the final design. In this procedure the internal forces (and therefore the elements $B_{j i}$ ) obtained from the LPP solution are assumed to be constant. In this way, the final design is only an approximation to the optimum of the fixed geometrical problem when its precision is dependent on the elements $B_{i j}$.

\subsection{Functions of linearized displacements}

Furthermore, it is possible to linearize the displacement dependences. A first order expansion of the Taylor series about the solution point of the LPP, $\left\{\begin{array}{c}* \\ X\end{array}\right\},\{N\}^{*}$ gives

$$
D_{j}=\stackrel{*}{D}_{j}+\sum_{i=1}^{n} \frac{\partial D_{j}^{*}}{\partial X_{i}}\left(X_{i}-\stackrel{*}{X_{i}}\right)+\sum_{k=1}^{m} \frac{\partial \stackrel{*}{D}_{j}}{\partial N_{k}}\left(N_{k}-\stackrel{N}{*}_{k}\right) \leq D_{j}^{U}
$$


where $m$ denotes the number of redundant forces ( statical indeterminacy degree). For determination of the displacement derivatives, it can be noted that the internal forces $\{A\}$ are linear functions of $\{N\}$ (eqn ...). Also, a statically equivalent internal force system corresponding to the virtual external load $Q_{i}$ may be selected so that the forces $A_{j i}^{Q}$ are fixed. In this way, the displacement $D_{i}$ may be rendered in the form

$$
D_{j}=\sum_{i=1}^{n} \frac{b_{j i 0}+\sum_{k=1}^{m} b_{j i k} N_{k}}{X_{i}}
$$

where $b_{j i 0}$ and $b_{j i k}$ are constants. After differentiating with regard to $X_{h}$ and $N_{f}$, respectively, we get

$$
\begin{gathered}
\frac{\partial D_{j}^{*}}{\partial X_{h}}=\frac{b_{j h 0}+\sum_{k=1}^{m} b_{j h k} \stackrel{*}{N}_{k}^{*}}{X_{h}^{2}} \\
\frac{\partial D_{j}}{\partial N_{f}}=\sum_{i=1}^{n} \frac{b_{i j f}}{\stackrel{*}{X}_{i}}
\end{gathered}
$$

Adding the linearized displacement constraints to the LPP, we can tackle an amplified LP problem. The steps of linearization and solution of the modified LP problem may be repeated until reaching convergence.

In the present formulation both $\{X\}$ and $\{N\}$ are used as independent variables. It is interesting to note that eqn (21) is also valid if the compatibility conditions are taken into account (i.e. $N_{k}$ depend on $X_{i}$ ) because $\partial B_{j i} / \partial X_{i} \equiv 0$.

\subsection{Adopting the virtual-load system}

The internal-force system relevant to the virtual external load $Q_{i}$ is required to be statically equivalent to $Q_{j}$ only. In a statically indeterminate structure, the forces $A_{j i}^{Q}$ are not unique and various choices exist for the statically equivalent system. Selecting, for example, the system indicated in fig. 1 (with hinges supposed at intermediate supports), the displacement term of eqn (20) comes down to

$$
D_{j}=\frac{b_{j h 0}+b_{j h, h-1} N_{h-1}+b_{j h h} N_{h}}{X_{h}}
$$

in which $\mathrm{h}$ indicates the member on which the virtual load $Q_{j}$ is applied, and $\mathrm{N}_{h-1}$, $N_{h}$ are bending moments over the supports of the $h$ th member. Inserting this equation into the displacement constraint $D_{j} \leq D_{j}^{\mathrm{U}}$ and rearranging, the linear form will be gained 


$$
b_{j h 0}+b_{j h, h-1} N_{h-1}+b_{j h h} N_{h}-D_{j}^{U} X_{h} \leq 0 .
$$

That means, any displacement constraint of a continuous beam can be expressed as a linear function of three variables and added to the LPP.

a)

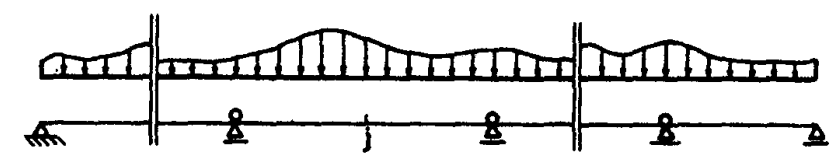

b)

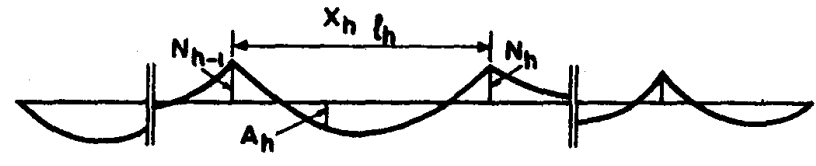

c)

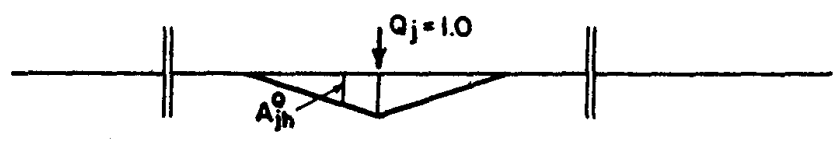

Figure 1: Continuous beam system.

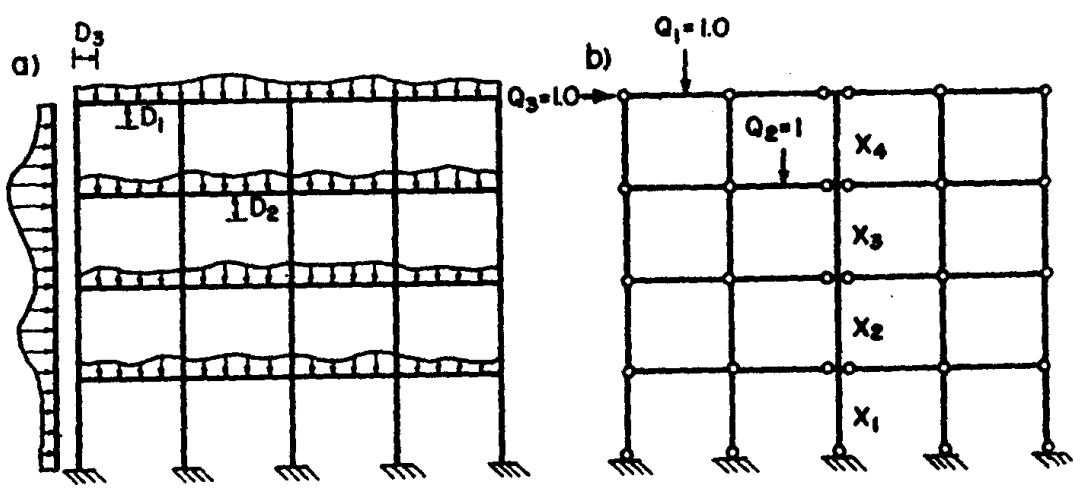

Figure 2: $\quad$ Multi-story frame.

Applying a similar procedure for the frame demonstrated in fig. 2(a) and adopting the equivalent system of fig. 2(b), the vertical displacement constraints (such as $D_{1} \leq D_{1}^{\mathrm{U}}$ or $D_{2} \leq D_{2}^{\mathrm{U}}$ ) can be expressed in the linear form of eqn (24). To obtain a linear horizontal displacement constraint $\left(D_{3} \leq D_{3}{ }^{\mathrm{U}}\right)$, it is necessary to suppose a linear relationship between the design variables of the middle column (fig. 2(b), only bending deformations are considered)

$$
X_{i}=\alpha_{i} X_{i} i=2,3,4 \text {. }
$$


The value of the coefficients $\alpha_{i}$ can be selected by virtue of the stress constraints. (The $\alpha_{i}$ can be derived, for example, from the LPP solution; subsequently, the linearized displacement constraint is added to the LPP and we get a revised solution.

\subsection{Example: statically determinated six-member lattice (fig. 3)}

Given quantities: the allowable stresses are $\left\{\sigma^{\mathrm{u}}\right\}=-\left\{\sigma^{\mathrm{L}}\right\}=150 \mathrm{MPa}$, the elasticity modulus $E=2.1 \cdot 10^{5} \mathrm{MPa}$, and the objective function $Z$ means the volume of material.

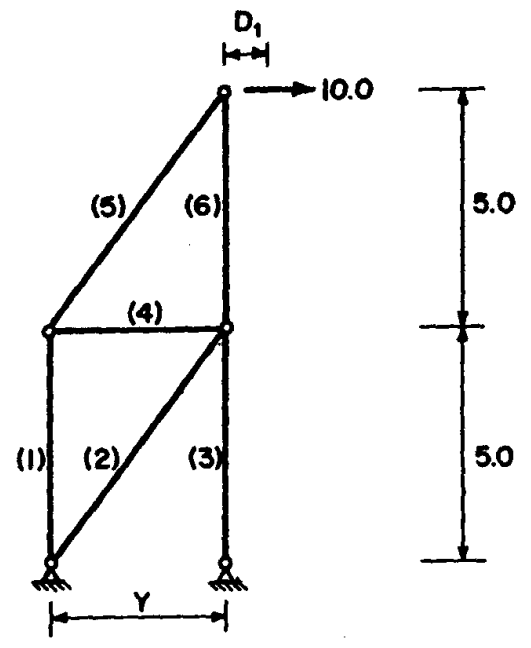

Figure 3: $\quad$ Six-member lattice.

The lattice is subject to a single horizontal load and the bar areas are selected being cross sectional design variables, with no side constraints described. The single variable $Y$ is subjected to the side constraints

$$
3 \leq Y \leq 10
$$

Two occurrences are analyzed to exemplify the sensitivity of $Z$ to alterations in $Y$.

Instance A The characteristic constraints are applied to stresses only

Instance B The displacements constraint

$$
D_{1} \leq 0.33
$$

is regarded as an additional demand $\left(D_{1}\right.$ represents the horizontal displacement at the top of the lattice).

Modification of min $Z$ with $Y$ for both instances is demonstrated in fig. 4. While large changes in $Y$ result in considerable variations of $\min Z$, it can be observed that the objective function is relatively not sensitive to changes in the geometric variable near the optimum. That is, a coarse grid of points (say, a minimum step size of $1.0 \mathrm{~m}$ ) could be assumed for $Y$.

As no redundant forces are included, there is no need to take into account the compatibility conditions, the problem statement is precision. 


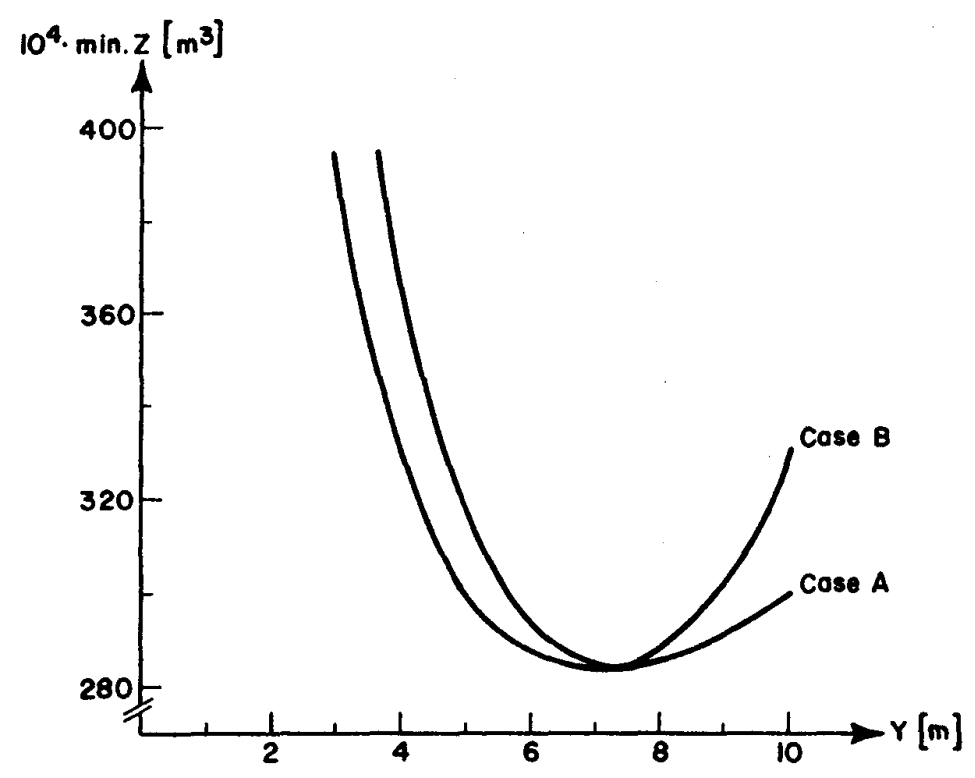

Figure 4: $\quad$ Variation of $\min Z$ with $Y$.

\section{Conclusion}

The evolution concepts and their choice in the optimization techniques of shell construction are pointed out. For effective optimization, the evolution strategy proved to be a competent means

To provide an efficacious tool for purposeful synthesis of structural geometry, it is appropriate to put together multilevel solution and approximation strategies. The multilevel statement combines simplified sub-optimization for member dimensions, reduction in the number of design variables optimized simultaneously, and improved convergence properties of the design problem. Disregarding provisionally the compatibility conditions, the AEP formulation is obtained. The latter qualifications do not appreciably influence the optimal geometry in a variety of construction, they are usually taken into account only for the final geometry obtained by the AEP solution. The AEP simulation yields a lower limit of the optimum and does not embrace multiple explicit analyses. In the given case, a single analysis is adequate to assess the final optimum. To improve the method of solution, the number of independent variables can be reduced by means of variable linking.

The phase of optimization of cross section for a fixed geometry uses plenty of the computational funds up, accordingly, it is fundamental to employ effective approaches at this period.

The interpretations stated simplify the method of geometric optimization.

According to paper [1], theoretical and numerical procedures for the subject matter of shell roofs were applied to one bionics roof construction made of laminated wood, erected in the town Brezno, Slovakia. 
86 Computer Aided Optimum Design in Engineering X

\section{Acknowledgement}

The author gratefully acknowledges the financial support of the presented research by the Grant Agency of the Czech Republic (project No. 103/06/1382).

\section{References}

[1] Tesár, A., Dynamic optimization for geometry of bionics shell roofs subjected to aimed snow skidding. Building Research Journal, 54(1), pp. 53-66, 2006.

[2] Rechenberg, J., Evolutionstrategie. Reihe Problemata 15, F. Fromman Verlag, Stuttgart-Bad Cannstadt, 1977.

[3] Kirsch, U., Synthesis of structural geometry using approximate concepts. Computers Structures, 15(3), pp. 305-314, 1982. 